\title{
Diseño de un ambiente de aprendizaje blended learning como propuesta de innovación educativa en la Universidad de la Sierra Juárez
}

Design of a Blended Learning Environment as a Proposal for Educational Innovation in the Universidad de la Sierra Juárez

Desenho de um ambiente de blended learning como proposta de inovação educacional na Universidad de la Sierra Juárez

\author{
Florentino Mendez Gijon \\ Universidad de la Sierra Juárez, México \\ Universidad Autónoma de Querétaro, México \\ fmendez@unsij.edu.mx \\ https://orcid.org/0000-0003-1377-6441
}

Magda Concepción Morales Barrera Centro Interdisciplinario de Investigación y Docencia en Educación Técnica (Ciidet), México mmorales@ciidet.edu.mx https://orcid.org/0000-0003-2329-4665 


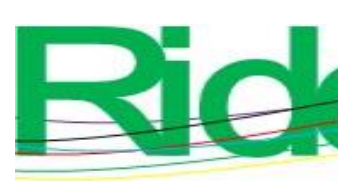

Revista Iberoamericana para la
Investigación y el Desarrollo Educativo
ISSN $2007-7467$

Evaluada como pedagógicamente exitosa donde ha sido implementada, la modalidad blended learning combina la enseñanza presencial con la virtual. En este trabajo se presenta una propuesta de diseño de un ambiente de aprendizaje para esta modalidad para el contexto educativo de la Universidad de la Sierra Juárez (Unsij). El desarrollo del ambiente se realizó con la metodología de investigación basada en el diseño (IBD), que permitió generar un prototipo de enfoque socioconstructivista adecuado al contexto universitario de la Unsij. En un primer momento, se realizó el diagnóstico del contexto para, entre otras cosas, analizar el mapa curricular de los programas educativos, valorar la infraestructura tecnológica con la que cuenta la universidad e identificar las actitudes y competencias digitales docentes. Como parte de los resultados, la universidad cuenta con recursos digitales/tecnológicos que sirven de apoyo a la docencia, pero no con una plataforma digital de gestión del aprendizaje (LMS). Asimismo, existe una actitud favorable de los profesores para usar las tecnologías de la información y la comunicación (TIC) en su práctica docente y un nivel de competencia intermedio en el conocimiento y uso en diversas actividades, pero un nivel bajo en el uso de estas herramientas para el diseño de actividades didácticas. Por último, el modelo educativo de la institución es de tipo presencial a tiempo completo, con planes de estudio que se agrupan en dos periodos semestrales y uno de verano; en los periodos semestrales se cursan cinco materias a las que los estudiantes deben dedicar horas de estudio con docente (presencial en aula) y otras independientes. La información obtenida ayudó al diseño de un ambiente de aprendizaje para la modalidad blended learning, que si bien continúa a prueba y se mantiene en proceso de evaluación y seguimiento, hasta este momento ha ayudado a mejorar la práctica docente en un ambiente mediado con TIC.

Palabras clave: ambiente de aprendizaje, educación superior, enseñanza mixta, innovación educativa, tecnología educativa. 


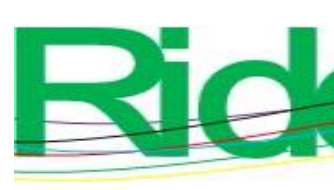

Revista Iberoamericana para la Investigación y el Desarrollo Educativo ISSN $2007-7467$

\section{Abstract}

Evaluated as pedagogically successful where it has been implemented, the blended learning modality combines face-to-face with virtual teaching. This work presents a proposal for the design of a learning environment for this modality for the educational context of the Universidad de la Sierra Juárez (UNSIJ, by its acronym in Spanish). The development of the environment was carried out with a design-based research (DBR), which allowed to generate a prototype of a socio-constructivist approach appropriate to the UNSIJ context. At first, the context diagnosis was carried out in order, among other things, to analyze the curricular map of educational programs, assess the technological infrastructure that the university has, and identify teaching attitudes and digital skills. As part of the results, the university has digital / technological resources that support teaching, but not a digital learning management platform (LMS). Likewise, there is a favorable attitude of teachers to use information and communication technologies (ICT) in their teaching practice and an intermediate level of competence in knowledge and use in various activities, but a low level in the use of these tools for the design of didactic activities. The information obtained helped to design a learning environment for the blended learning modality, which although it continues to be tested and remains in the evaluation and monitoring process, up to now it has helped to improve practice teaching in an ICT-mediated environment.

Keywords: learning environment, higher education, blended learning, educational innovation, educational technology.

\section{Resumo}

Avaliada como pedagogicamente bem-sucedida onde foi implementada, a modalidade de ensino híbrido combina o ensino presencial com o ensino virtual. Este trabalho apresenta uma proposta para o desenho de um ambiente de aprendizagem para esta modalidade para o contexto educacional da Universidade da Serra Juárez (Unsij). O desenvolvimento do ambiente foi realizado com a metodologia de pesquisa baseada no design (IBD), o que permitiu gerar um protótipo de uma abordagem sócio-construtivista adequada ao contexto universitário Unsij. Num primeiro momento, foi realizado o diagnóstico de contexto com o objetivo, entre outras coisas, de analisar o mapa curricular dos programas educativos, avaliar a infraestrutura tecnológica de que dispõe a universidade e identificar atitudes docentes e competências digitais. Como parte dos resultados, a universidade possui 


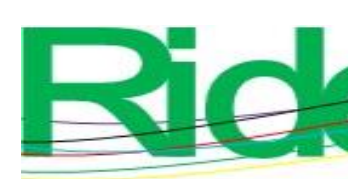

Revista Iberoamericana para la Investigación y el Desarrollo Educativo ISSN $2007-7467$

estrategias fallidas de aprendizaje que les impiden desarrollarse de forma exitosa en su formación profesional y después de ella (Organización para la Cooperación y el Desarrollo Económicos [OCDE], 2016).

Estas problemáticas son enfrentadas en el aula por el profesor, quien, en la mayoría de los casos, no tiene una suficiente formación docente como para ser capaz de atenderlas. Esta carencia, sin embargo, puede ser vista como un área de oportunidad y un campo fértil para innovar y proporcionar alternativas que incluyan herramientas que puedan guiar a los estudiantes inmersos en este contexto globalizado, tecnificado y con las características heredadas de los niveles educativos previos. Dichas herramientas tienen que estar enmarcadas en el contexto donde se lleva a cabo el proceso de enseñanza-aprendizaje, es decir, deben tomar en cuenta los rasgos particulares de los profesores y estudiantes, el modelo educativo y la infraestructura tecnológica (Delors, 1996).

Una parte de estas herramientas docentes son los recursos técnicos con los que se apoya el proceso de enseñanza-aprendizaje y donde las TIC juegan un rol de suma importancia, ya que permiten procesar, transmitir y almacenar de forma digital enormes cantidades de información con diferentes fines, siempre y cuando se cuente con dispositivos electrónicos e Internet. El Internet, como es bien sabido, es uno de los desarrollos tecnológicos más disruptivos de los últimos tiempos. En 1989, potenció la aparición de la web 1.0, la cual ha ido evolucionando con el paso de los años y los avances tecnológicos; ahora se habla de web 2.0, web 3.0 e incluso web 4.0. A partir de la web 2.0 surgió la posibilidad de interactuar entre usuarios y contenidos, y permitió a aquellos ya no solo ser consumidores, sino también creadores de la información que se publicaba; surgieron también nuevos canales de comunicación: las redes sociales en línea, sitios colaborativos como las wikis, redes de colaboración académica y científica, por ejemplo, la Red Nacional de Educación e Investigación (RNEI), el comercio electrónico y, por supuesto, la educación en línea (e-learning), que retomó algunos de los fundamentos de la educación a distancia (Choudhury, 2014; Evans, 2011; Rodríguez y Espinoza, 2017).

Para el soporte tecnológico del e-learning surgieron desarrollos basados en el avance de la web como los sistemas de gestión de aprendizaje (LMS, por sus siglas en inglés) y los cursos masivos abiertos en línea (MOOC, por sus siglas en inglés). Estos desarrollos, en conjunto con los principios pedagógicos y las metodologías de diseño instruccional, se han ido adoptando en los sistemas educativos de enseñanza presencial; de este modo, han surgido nuevas formas que conjugan ambas modalidades de formación. Una 


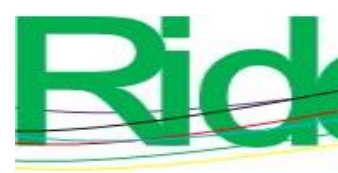

Revista Iberoamericana para la
Investigación y el Desarrollo Educativo
ISSN $2007-7467$

de estas es precisamente el blended learning, que combina la enseñanza en línea con la presencial para aprovechar las ventajas de cada una de ellas.

Por brindar la posibilidad de innovar la práctica educativa mediante la inclusión de las TIC en la enseñanza presencial, el blended learning es una modalidad que ha mostrado ser pedagógicamente exitosa (Siemens, Gašević y Shane, 2015). Para aprovechar sus ventajas, es necesario que esta combinación parta de una planeación cuidadosa que tome en cuenta diversos factores (pedagógicos, tecnológicos y contextuales) que conduzcan a un verdadero proceso de innovación educativa orientado a transformar o fortalecer el proceso de enseñanza-aprendizaje. En ese sentido, el profesor es uno de los actores principales, ya que con su práctica puede lograr formar estudiantes más comprometidos y activos en su proceso de aprendizaje y lograr cambiar la concepción de su función como docente: de ser un transmisor del conocimiento a guiar a los alumnos en la ruta de su capacitación (Necuzzi, 2013; Salinas, 2004).

En este trabajo se sostiene que una de las formas de lograr esta transformación es la puesta en práctica de estrategias pedagógicas enmarcadas en un ambiente de aprendizaje para una modalidad blended learning; definir actividades para que el alumnado se apropie de los conocimientos y el docente lo guíe en ese proceso, y dar la oportunidad al discente que sea él quien construya o reconstruya sus conocimientos relacionados con las materias que se abordan.

En una primera sección se describe el marco teórico del proyecto. Desde un enfoque constructivista, se aborda el blended learning. En una segunda parte se aborda la metodología de investigación basada en el diseño (IBD) que se siguió para el desarrollo de la propuesta. Este proceso se dividió en las etapas siguientes: análisis de la situación, desarrollo de las soluciones, implementación y validación y la producción de documentación y principios de diseño. Posteriormente se exponen los resultados del análisis del contexto universitario. Y finalmente, en las conclusiones y discusiones se realiza un contraste con otros trabajos y principios teóricos de la modalidad blended learning. 


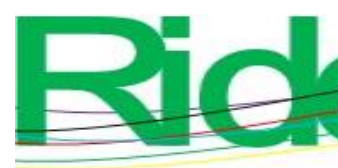

Revista Iberoamericana para la Investigación y el Desarrollo Educativo ISSN $2007-7467$

citado en Chadwick, 2001). En un ambiente mezclado, ese mentor puede ser el profesor, un compañero de clase o alguien externo al espacio físico donde se desarrolla el curso. Incluso, en un entorno digitalizado, ese experto puede ser un sistema informático con capacidad de atender dudas específicas, basado en información que previamente le ha sido cargada. Si se observa, en este contexto el profesor ya no es el poseedor del conocimiento, más bien es quien planea el proceso para que el aprendizaje tenga lugar.

Con base en estos planteamientos, en la siguiente expresión se sugiere la idea de constructivismo que sustenta la propuesta que se presenta en este trabajo:

\section{Socioconstructivismo $=$ Estructuras cognitivas $($ experiencias previas $)+$ Aprendizaje significativo + Contexto sociocultural}

En este sentido, las teorías sociocognitivas constructivistas y de aprendizaje significativo pueden servir de sustento para el diseño de tareas académicas centradas en el estudiante. Por un lado, el constructivismo cognitivo es la base del diseño de actividades que atienden las necesidades e intenciones de aprendizaje individual de los alumnos. Y a partir del constructivismo social se sugiere el diseño de actividades que favorezcan el aprendizaje colaborativo en el que los estudiantes pueden aprender de forma significativa y correcta unos de otros, por lo que el diseño social de un entorno formativo mezclado debe cumplir con características de seguridad, disponibilidad, usabilidad y contar con un conjunto de estrategias de comunicación virtual y presencial que motiven la comunicación entre los estudiantes (Wang, 2008). Por otro lado, este ambiente constructivista debe permitir al profesor guiar el proceso capacitador de los alumnos.

Estos planteamientos constructivistas pueden llevarse a la práctica en una modalidad blended learning, la cual, como ya se mencionado, ha mostrado efectividad en mejorar las prácticas pedagógicas, ya que ha permitido introducir estrategias de aprendizaje activo, de igual a igual, centradas en el estudiante y en el trabajo colaborativo, al mismo tiempo que ha brindado la posibilidad de alcanzar altos niveles de capacitación a través del discurso crítico y pensamiento reflexivo con el uso de la comunicación síncrona en las clases presenciales y la comunicación asíncrona a través de canales de comunicación virtuales, los cuales, por su parte, gracias a los avances tecnológicos de la Web, las plataformas LMS y los servicios en la nube, brindan la oportunidad de crear comunidades de investigación (Garrison y Kanuka, 2004; Graham, 2006; Güzer y Caner, 201). 


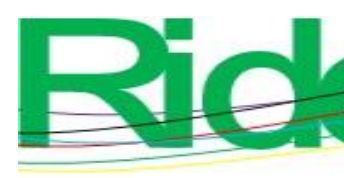

\section{Revista Iberoamericana para la Investigación y el Desarrollo Educativo ISSN $2007-7467$}

Aunado a lo anterior, la modalidad blended learning puede tomar diferentes formas en su implementación. La forma dependerá, entre otros factores, de los propósitos que se persiguen para llevar a cabo su puesta en marcha, al igual que de los recursos tecnológicos disponibles de la institución, estudiantes y profesores y los tipos de contenido que se mezclarán. Estos aspectos ayudarán a determinar el tipo de actividades de aprendizaje que pueden implementarse en el ambiente blended learning, lo que definirá si las actividades serán en formato presencial, en línea o combinadas. Este tipo de tareas pueden ser de comunicación, colaboración, presentaciones multimedia o de estudio independiente; en conjunto permiten una configuración como la mostrada en la figura 1.

Figura 1. Ambiente blended learning

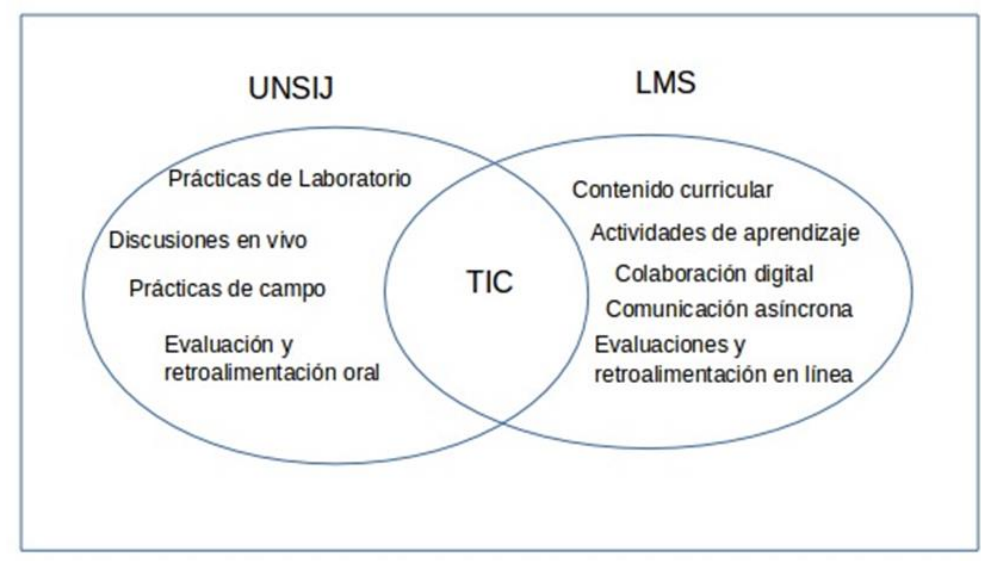

Fuente: Elaboración propia con base en Graham (2006)

La combinación de recursos tecnológicos y herramientas tecnopedagógicas dan lugar a lo que Garrison y Vaughan (2008) consideran una integración cuidadosa de las experiencias de aprendizaje presencial con experiencias de aprendizaje en línea o elearning para procurar la redefinición de la enseñanza centrada en el profesor hacia un enfoque que posiciona más la labor docente como guía del aprendizaje de los estudiantes. En este esquema, las sesiones de enseñanza presencial son las que se desarrollan en el espacio físico de la institución para aprovechar las ventajas de la interacción cara a cara; por ejemplo, realizar retroalimentación de forma inmediata mediante sesiones de discusión, aclarar dudas, realizar prácticas de laboratorio con equipos físicos, salidas de campo, visitas a empresas, estancias profesionales, entre muchas otras.

Todas estas actividades en un ambiente mediado con TIC se centralizan para darles orden y seguimiento a través de un LMS, donde se hace el despliegue del contenido pedagógico. Este contenido incluye materiales, recursos, herramientas y actividades que 


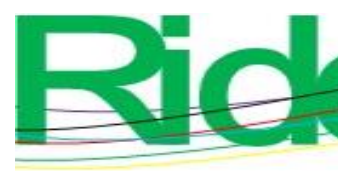

Revista Iberoamericana para la
Investigación y el Desarrollo Educativo
ISSN $2007-7467$

pueden ser consultados de forma ubicua por los estudiantes dentro y fuera de las aulas. Los LMS permiten también crear entornos de aprendizaje dinámicos y con una rica interacción social, y hacer uso de sus diferentes herramientas estandarizadas que brindan la posibilidad de fomentar la comunicación y el aprendizaje colaborativo a través de canales de comunicación: foros de discusión y sección de mensajería síncrona y asíncrona. En lo que respecta al seguimiento del curso, los profesores pueden realizar el despliegue de sus materias apegado al modelo educativo institucional deseado, así como monitorear el avance de los estudiantes con las diferentes herramientas de evaluación y libros de calificación. Todos estos componentes tecnopedagógicos aquí se entienden como parte de un sistema de e-learning que, combinado con la enseñanza presencial, conduce a un ambiente blended learning.

\section{Metodología}

Para la elaboración de la propuesta se siguió la metodología IBD, que permite mejorar el diseño implementado y la generación de pautas para la ejecución de diseños educativos en situaciones con condiciones similares, más que la replicación de las implementaciones realizadas. Toda investigación de diseño tiene como uno de sus propósitos la producción de contribuciones teóricas, ya sea para precisar, extender, convalidar o modificar teoría existente o generar nueva teoría (de Benito y Salinas, 2016).

El diseño que aquí se presenta se divide en un conjunto de fases que se observan en la figura 2. Como se puede apreciar, se retoman las propuestas de de Benito y Salinas (2016), quienes establecen las etapas puestas a continuación: definición del problema, diseño, desarrollo, implementación y evaluación; así como la de Reeves (2000), que divide el proceso de investigación en un conjunto de interacciones que parte del análisis de la situación/definición del problema y continúa con el desarrollo de soluciones de acuerdo con un fundamentación teórica, la implementación, la validación, la producción de documentación y los principios de diseño. 


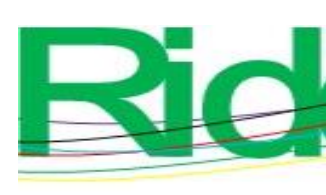

\section{Revista Iberoamericana para la Investigación y el Desarrollo Educativo ISSN $2007-7467$}

Figura 2. Fases de la investigación

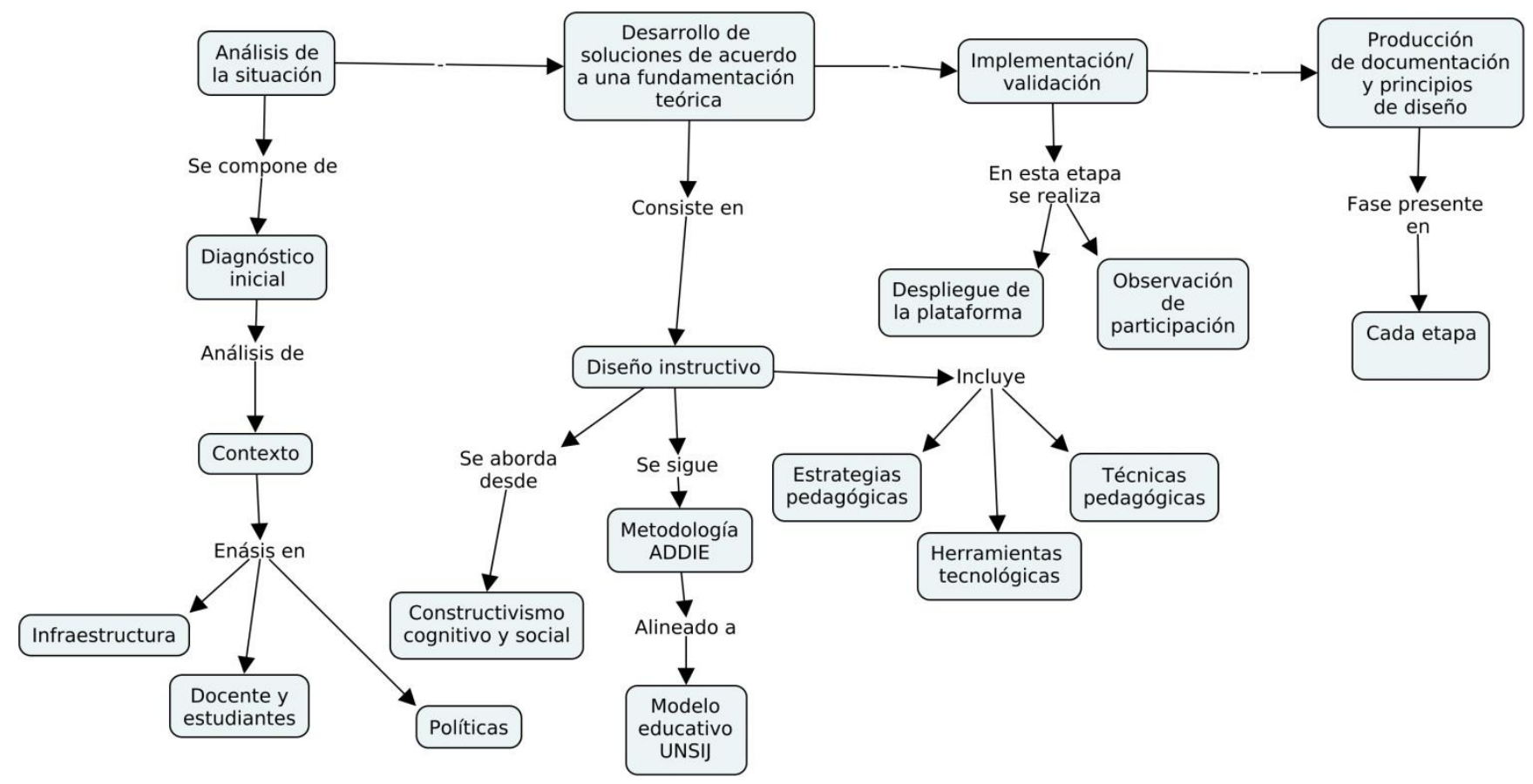

Fuente: Elaboración propia con base en Reeves (2000)

La fase de diagnóstico tuvo como finalidad evaluar la pertinencia de la implementación del ambiente de aprendizaje blended learning, por lo que, en una de las actividades, se recabó información sobre la disponibilidad de la infraestructura TIC. Para ello se realizó una búsqueda documental a partir de los inventarios disponibles en el Departamento de Recursos Materiales de la universidad seleccionada, a saber, la Universidad de la Sierra Juárez (Unsij), Oaxaca, México. También se entrevistó al jefe del Departamento de Red de Cómputo de la institución, responsable de administrar y gestionar la infraestructura de TIC, al igual que al jefe de carrera de la licenciatura en Informática, que es el encargado de las salas de cómputo institucionales.

Otra de las actividades de la fase de diagnóstico consistió en recabar información sobre las competencias digitales docentes por medio del cuestionario de Tourón, Martín, Navarro, Prada e Íñigo (2018), que retoma las categorías de análisis del modelo de competencias digitales docentes del Instituto Nacional de Tecnologías Educativas y Formación del Profesorado [Intef] (2017), además de las actitudes docentes frente a las TIC mediante el cuestionario propuesto por Tejedor, García y Prada (2009). Ambos instrumentos fueron aplicados a un total de ocho docentes. Igualmente, se recabó información del nivel de acceso a TIC de los estudiantes de la carrera de la licenciatura en Informática; un total de 35 personas, de entre 18 y 23 años de edad, con quienes se inició la 


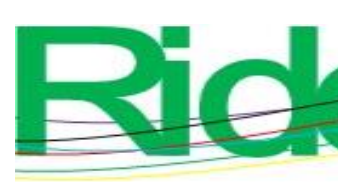

Revista Iberoamericana para la
Investigación y el Desarrollo Educativo
ISSN $2007-7467$

prueba del ambiente de aprendizaje blended learning, a través del cuestionario que se sugiere Intel Corporation (2011).

Otra de las acciones consistió en revisar el modelo educativo de la Unsij centrando la atención en la estructura curricular de los planes de estudio de las diferentes licenciaturas que ahí se imparten.

En la fase de desarrollo de soluciones, se llevó a cabo el diseño instructivo para el ambiente blended learning adecuado al modelo educativo y específicamente a la estructura curricular de la Unsij, siguiendo la metodología de análisis, diseño, desarrollo, implementación y evaluación (Addie) y bajo los preceptos teóricos del socioconstructivismo. Lo anterior con el fin de plantear un modelo de ambiente de aprendizaje de este tipo que le permita al docente emplearlo en el diseño de su instrucción, y así modificar o mejorar su práctica para transitar de un modelo de enseñanza centrado en el profesor hacia un entorno donde el estudiante asuma un papel activo en su proceso de aprendizaje. Este modelo se puso en marcha en la fase de implementación y validación, haciendo uso de una plataforma LMS Moodle, que se desplegó dentro de la infraestructura universitaria y permitió recabar información de cómo los docentes adoptaron el modelo mediante el seguimiento de las actividades docentes en la plataforma LMS.

\section{Resultados}

\section{Diagnóstico}

De los resultados analizados en la fase de diagnóstico, se encuentra que las condiciones actuales del contexto de la Unsij son adecuadas para poder adoptar la modalidad blended learning, ya que desde el plano legal, en el artículo dos del Reglamento del Personal Docente de la citada institución, se establece como una de las funciones sustantivas del personal académico de la universidad el impartir educación bajo el principio de libertad de cátedra. Al respecto, siguiendo a Seara (2010): "La libertad de cátedra solo tiene validez en el marco de los planes de estudios vigentes y de modo que el programa de la materia se cubra adecuadamente" (p. 44). Por otro lado, los planes de estudio de la Unsij se estructuran con base en asignaturas agrupadas en áreas de estudio; cinco asignaturas son cursadas en un semestre académico, dividido en tres periodos parciales de cinco semanas y un periodo ordinario de dos semanas. Una asignatura puede tener desde seis, ocho o diez créditos. Un crédito es la estimación del tiempo de trabajo académico para el estudiante, y 


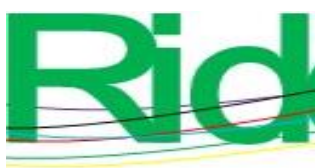

2

Revista Iberoamericana para la Investigación y el Desarrollo Educativo

ISSN $2007-7467$

asignaturas. Hacen un uso moderado de herramientas para la creación de contenido pedagógico digital, y no fomentan esta actividad en las tareas de los estudiantes. Ocurre algo similar en lo concerniente al uso seguro de las TIC. En promedio también muestran un nivel de competencia intermedia en la resolución de problemas básicos de dispositivos y software, sin embargo, los datos recabados arrojan que hace falta fomentar en el personal docente un mayor nivel de colaboración y ayuda mutua en el entorno digital. En la tabla 2 se muestra un resumen de las diferentes áreas analizadas con respecto a las competencias digitales docentes.

Tabla 2. Resumen de categorías y nivel de uso y conocimiento en las competencias digitales docente de las y los profesores de la Unsij

\begin{tabular}{|l|c|}
\hline \multicolumn{1}{|c|}{ Áreas } & Nivel de conocimiento y uso \\
\hline Información y alfabetización informacional & Intermedio \\
\hline Comunicación y colaboración & Intermedio \\
\hline Creación de contenidos digitales & Básico \\
\hline Seguridad & Básico \\
\hline Resolución de problemas & Intermedio \\
\hline
\end{tabular}

Fuente: Elaboración propia

En lo que refiere al equipamiento TIC de la universidad, en la tabla 3 se presenta un resumen de las instalaciones y equipo relacionado con este rubro propiedad de la universidad, y en la tabla 4 se pueden observar los servicios de red que se ofertan en la infraestructura universitaria, la cual también brinda servicios de conectividad a Internet de banda ancha $(100 \mathrm{Mb} / \mathrm{s})$ a través de computadoras institucionales o equipos personales a todos los profesores y estudiantes. 


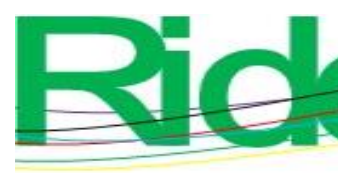

\section{Revista Iberoamericana para la Investigación y el Desarrollo Educativo ISSN $2007-7467$}

\section{Diseño instructivo}

La propuesta se aborda desde una perspectiva socioconstructivista para la integración de las TIC en el proceso de enseñanza-aprendizaje (Wang, 2008). Para el abordaje de las asignaturas, se propone un esquema de distribución de los tiempos a partir del número de créditos descritos en el plan de estudios de la materia y teniendo en cuenta que en el semestre un estudiante debe cursar cinco asignaturas de manera simultánea, correspondientes a las áreas disciplinares de su formación, y una de aprendizaje de lengua extranjera. Este esquema se muestra en la tabla 1.

Los periodos para el desarrollo de las asignaturas se definen en el calendario escolar, que, como ya se mencionó, divide el semestre académico en tres periodos parciales y un ordinario. Para cada uno de los parciales se establece un tiempo de 50 horas de trabajo académico divididas en cinco semanas por cada materia cursada, de las que 25 horas deben ser HED y el resto HEI. Para el periodo ordinario se consideran cinco HED y el resto de HEI divididas en dos semanas. Esta distribución se resume en la tabla 5.

Tabla 5. Distribución de tiempos por parcial y por ordinario

\begin{tabular}{|c|c|c|c|c|c|c|c|c|c|}
\hline \multirow[b]{2}{*}{$\begin{array}{l}\text { Crédito } \\
\text { s de la } \\
\text { materia }\end{array}$} & \multirow[b]{2}{*}{$\begin{array}{c}\text { Parciale } \\
\text { s }\end{array}$} & \multirow[b]{2}{*}{$\begin{array}{c}\text { Ordinar } \\
\text { io }\end{array}$} & \multirow[b]{2}{*}{$\begin{array}{c}\text { Semanas } \\
\text { por } \\
\text { parcial }\end{array}$} & \multirow[b]{2}{*}{$\begin{array}{l}\text { Semanas } \\
\text { por } \\
\text { ordinario }\end{array}$} & \multicolumn{5}{|c|}{ Total de horas de trabajo } \\
\hline & & & & & $\begin{array}{l}\text { HED } \\
\text { parcial }\end{array}$ & $\begin{array}{c}\text { HEI } \\
\text { parcial }\end{array}$ & $\begin{array}{c}\text { HED } \\
\text { Ordinar } \\
\text { io }\end{array}$ & $\begin{array}{c}\text { HEI } \\
\text { Ordinario }\end{array}$ & Total de horas \\
\hline 10 & 3 & 1 & 5 & 2 & 25 & 25 & 5 & 5 & 160 \\
\hline 8 & 3 & 1 & 5 & 2 & 25 & 14 & 5 & 6 & 128 \\
\hline 6 & 3 & 1 & 5 & 2 & 25 & 4 & 5 & 4 & 96 \\
\hline
\end{tabular}

Fuente: Elaboración propia

Respecto a la evaluación en cada asignatura, se destina un peso de $50 \%$ para los tres periodos parciales y el otro $50 \%$ para el ordinario, y queda a criterio del profesor establecer la ponderación para cada periodo parcial de acuerdo con las actividades de aprendizaje propuestos. Los planes de estudio sugieren en cada uno de los parciales aplicar un examen escrito y complementarlo con las demás actividades académicas.

En la planeación para cada curso se propone una secuencia didáctica dividida en tres momentos: inicio, desarrollo y cierre; a excepción del ordinario, periodo en el que únicamente se considera un momento de cierre (por ser corto). A cada uno de estos se les 


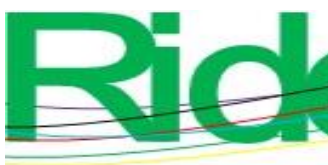

Revista Iberoamericana para la Investigación y el Desarrollo Educativo ISSN $2007-7467$

asigna un tiempo determinado de HED y HEI, dependiendo del tipo de actividad, como se observa en la tabla 6. Con respecto a la asignación de las horas por cada actividad, se precisa que estas dependerán de la asignatura y de las características de los estudiantes, por lo que existe flexibilidad por parte del profesor en la distribución de los tiempos. Asimismo, este esquema también puede distribuirse por periodos semestrales; no obstante, por las mismas restricciones de los planes de estudio para la evaluación de los alumnos, la distribución que aquí se muestra es la más adecuada para este contexto universitario.

Tabla 6. Propuesta de distribución de sesiones, momentos, semanas y horas de trabajo por asignatura

\begin{tabular}{|c|c|c|c|c|c|c|c|c|c|c|c|c|c|c|c|c|}
\hline $\mathrm{S} 1$ & $\mathrm{~S} 2$ & S3 & $\mathrm{S} 4$ & S5 & S6 & S7 & S8 & S9 & S10 & S11 & $\mathrm{S} 12$ & S13 & S14 & $\mathrm{S} 15$ & S16 & S17 \\
\hline \multicolumn{17}{|c|}{ Momentos y periodos } \\
\hline \multicolumn{5}{|c|}{ Parcial 1} & \multicolumn{5}{|c|}{ Parcial 2} & \multicolumn{5}{|c|}{ Parcial 3} & \multicolumn{2}{|c|}{ Ordinario } \\
\hline $\mathrm{I}$ & $\mathrm{D}$ & $\mathrm{D}$ & $\mathrm{D}$ & $\mathrm{C}$ & $\mathrm{I}$ & $\mathrm{D}$ & $\mathrm{D}$ & $\mathrm{D}$ & $\mathrm{C}$ & $\mathrm{I}$ & $\mathrm{D}$ & $\mathrm{D}$ & $\mathrm{D}$ & $\mathrm{C}$ & & $\mathrm{C}$ \\
\hline \multicolumn{5}{|c|}{ HED } & \multicolumn{5}{|c|}{ HED } & \multicolumn{5}{|c|}{ HED } & \multicolumn{2}{|c|}{ HED } \\
\hline 5 & \multicolumn{3}{|c|}{15} & 5 & 5 & \multicolumn{3}{|c|}{15} & 5 & 5 & \multicolumn{3}{|c|}{15} & 5 & \multicolumn{2}{|c|}{5} \\
\hline \multicolumn{5}{|c|}{ HEI 10 Créditos } & \multicolumn{5}{|c|}{ HEI 10 Créditos } & \multicolumn{5}{|c|}{ HEI 10 Créditos } & \multicolumn{2}{|c|}{ HEI $10 \mathrm{C}$} \\
\hline 5 & \multicolumn{3}{|c|}{15} & 5 & 5 & \multicolumn{3}{|c|}{15} & 5 & 5 & \multicolumn{3}{|c|}{15} & 5 & \multicolumn{2}{|c|}{15} \\
\hline \multicolumn{5}{|c|}{ HEI 8 Créditos } & \multicolumn{5}{|c|}{ HEI 8 Créditos } & \multicolumn{5}{|c|}{ HEI 8 Créditos } & \multicolumn{2}{|c|}{ HEI $8 \mathrm{C}$} \\
\hline 2 & \multicolumn{3}{|c|}{10} & 2 & 2 & \multicolumn{3}{|c|}{10} & 2 & 2 & \multicolumn{3}{|c|}{10} & 2 & \multicolumn{2}{|c|}{6} \\
\hline \multicolumn{5}{|c|}{ HEI 6 Créditos } & \multicolumn{5}{|c|}{ HEI 6 Créditos } & \multicolumn{5}{|c|}{ HEI $6 \mathrm{C}$} & \multicolumn{2}{|c|}{ HEI $6 \mathrm{C}$} \\
\hline 1 & \multicolumn{3}{|c|}{2} & 1 & 1 & \multicolumn{3}{|c|}{2} & 1 & 1 & & 2 & & 1 & & 4 \\
\hline
\end{tabular}

Fuente: Elaboración propia

El tiempo de trabajo por momento de aprendizaje servirá para estimar las horas de trabajo para el profesor y el estudiante, de acuerdo con el momento y el tipo de actividad. En la tabla 7 se presenta un bosquejo considerando los créditos de la materia para un periodo parcial. 


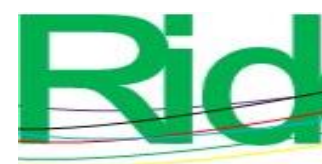

Revista Iberoamericana para la Investigación y el Desarrollo Educativo ISSN 2007 - 7467

Tabla 7. Lista de actividades por momento, periodo y créditos de la asignatura

\begin{tabular}{|c|c|c|c|c|c|c|c|}
\hline \multirow[t]{2}{*}{ Actividades } & \multirow[t]{2}{*}{ Momento } & \multicolumn{2}{|c|}{$\begin{array}{l}\text { Parcial de } 10 \\
\text { créditos }\end{array}$} & \multicolumn{2}{|c|}{$\begin{array}{c}\text { Parcial de } 8 \\
\text { créditos }\end{array}$} & \multicolumn{2}{|c|}{ Parcial de 6 créditos } \\
\hline & & HED & HEI & HED & HEI & HED & HEI \\
\hline $\begin{array}{l}\text { Recuperación de saberes } \\
\text { previos }\end{array}$ & Inicio & 2 & 3 & 2 & 2 & 2 & 1 \\
\hline Actividad de motivación & Inicio & 3 & 2 & 3 & 2 & 3 & 1 \\
\hline $\begin{array}{l}\text { Construcción de } \\
\text { conocimientos }\end{array}$ & Desarrollo & 7 & 8 & 7 & 3 & 7 & 1 \\
\hline $\begin{array}{l}\text { Aplicación de } \\
\text { conocimientos } \\
\text { (desarrollo de } \\
\text { habilidades) }\end{array}$ & Desarrollo & 8 & 7 & 8 & 3 & 8 & 1 \\
\hline Evaluación & Cierre & 5 & 5 & 5 & 4 & 5 & 1 \\
\hline
\end{tabular}

Fuente: Elaboración propia

En la tabla 8 se muestra una propuesta de actividades para una asignatura de 10 créditos partiendo de la distribución de tiempos y actividades sugeridas de la tabla 8 . 


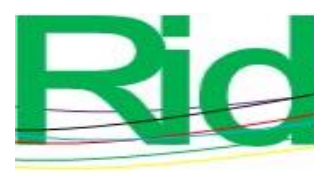

Figura 3. Formato de actividad blended

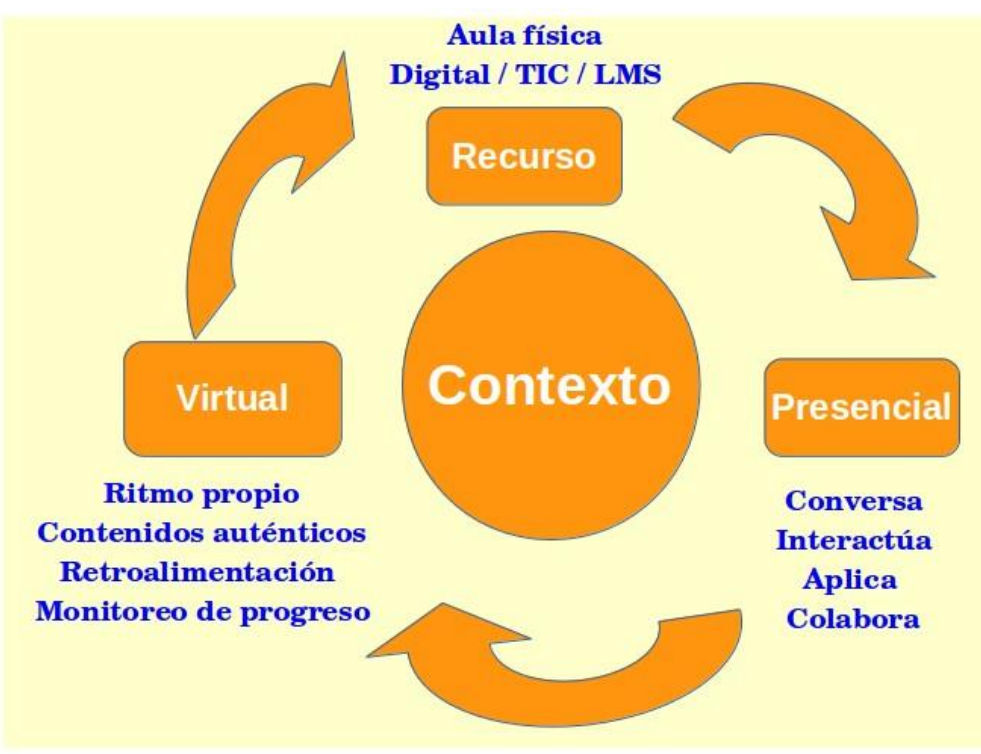

Fuente: Elaboración propia

La estructura de cada periodo está compuesta de por lo menos tres momentos y un conjunto de recursos. En cada uno de ellos se plantean un conjunto estrategias que le permiten al profesor guiar el proceso de aprendizaje de los estudiantes y donde el rol del docente se modifica de acuerdo con el momento. Los recursos pueden ser de elaboración propia o seleccionados de fuentes externas. Como sea que fuere, deben cumplir con las características de rigor científico, como lo expresa Florez (1997), y adecuados a los objetivos de aprendizaje planteados por el plan de estudios de cada asignatura. En la figura 4 se muestra el bosquejo de un periodo parcial. 

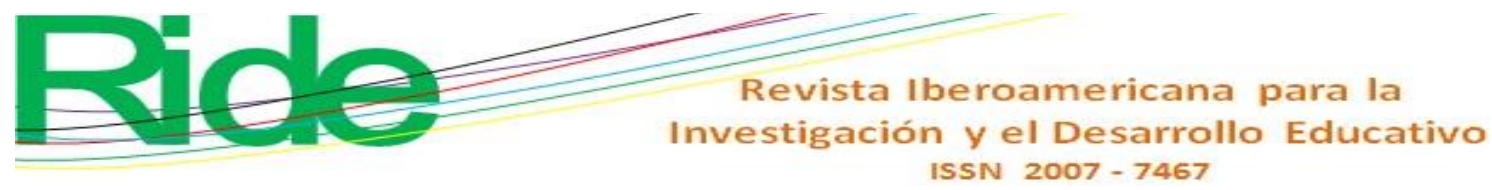

Figura 4. Estructura de un periodo

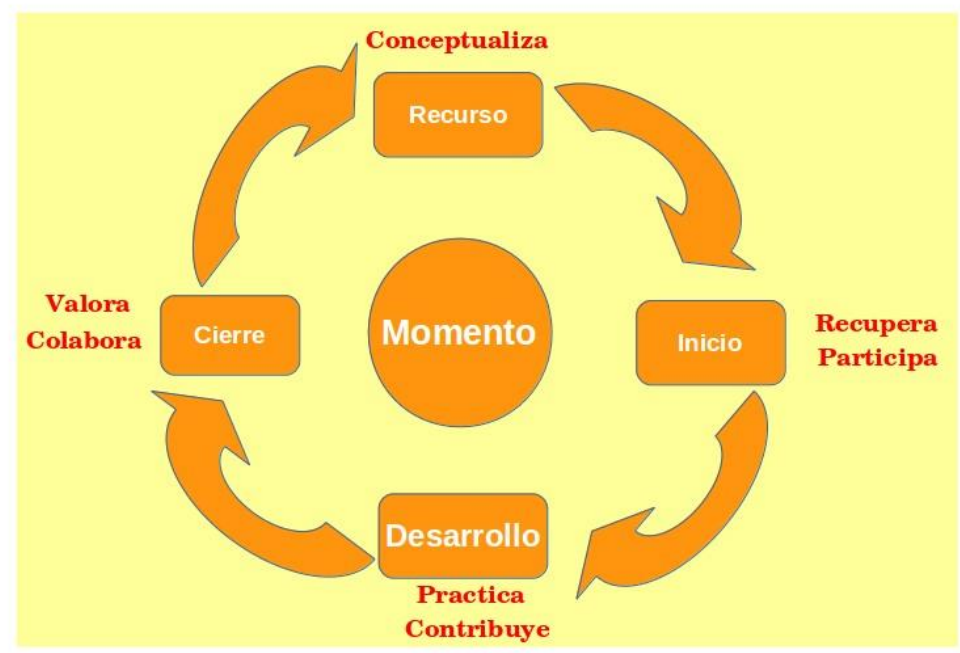

Fuente: Elaboración propia

La estructura semestral de una asignatura se observa en la figura 5 y está integrada por tres periodos parciales y un periodo ordinario, independientemente de los créditos que contenga, y en esta se engloban los recursos y actividades distribuidas en una modalidad blended learning, de acuerdo con la naturaleza de la asignatura y los objetivos de aprendizaje.

Figura 5. Estructura de un semestre escolar

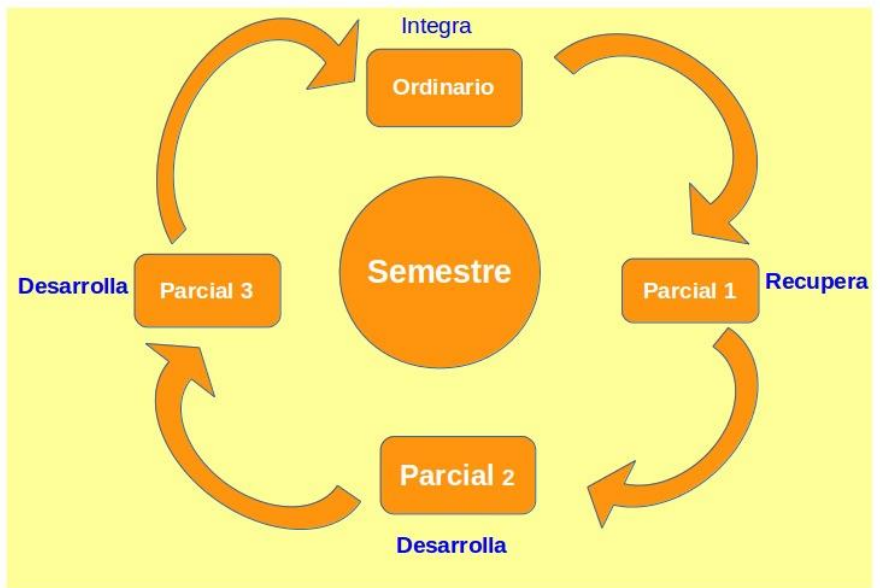

Fuente: Elaboración propia 

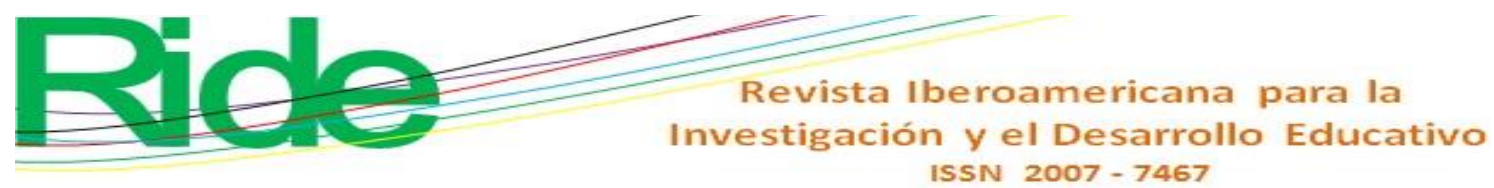

Este modelo se implementó en el segundo semestre del ciclo escolar 2018-2019 en dos asignaturas de la licenciatura en Informática de la Unsij. Para el despliegue virtual, se utilizó Moodle, que es un sistema web que se distribuye bajo los términos de la Licencia Pública General GNU. Este LMS brindó la posibilidad de organizar un curso en tópicos, y en cada uno de ellos se pudieron integrar actividades y recursos para guiar a los estudiantes en la ruta de su aprendizaje (ver figura 6). En este caso, cada tópico consistió de un periodo académico. El curso se dividió en tres periodos parciales y uno ordinario. En cada uno se desplegaron actividades de aprendizaje, foros de discusión, actividades de evaluación, recursos informacionales, así como el seguimiento de los estudiantes, apegándose a los tiempos planteados en los programas de estudio de cada asignatura y a los principios del socioconstructivismo (Aristizábal y Dieste, 2011; Moodle, 2019)

Figura 6. Portada de un curso desplegado en Moodle

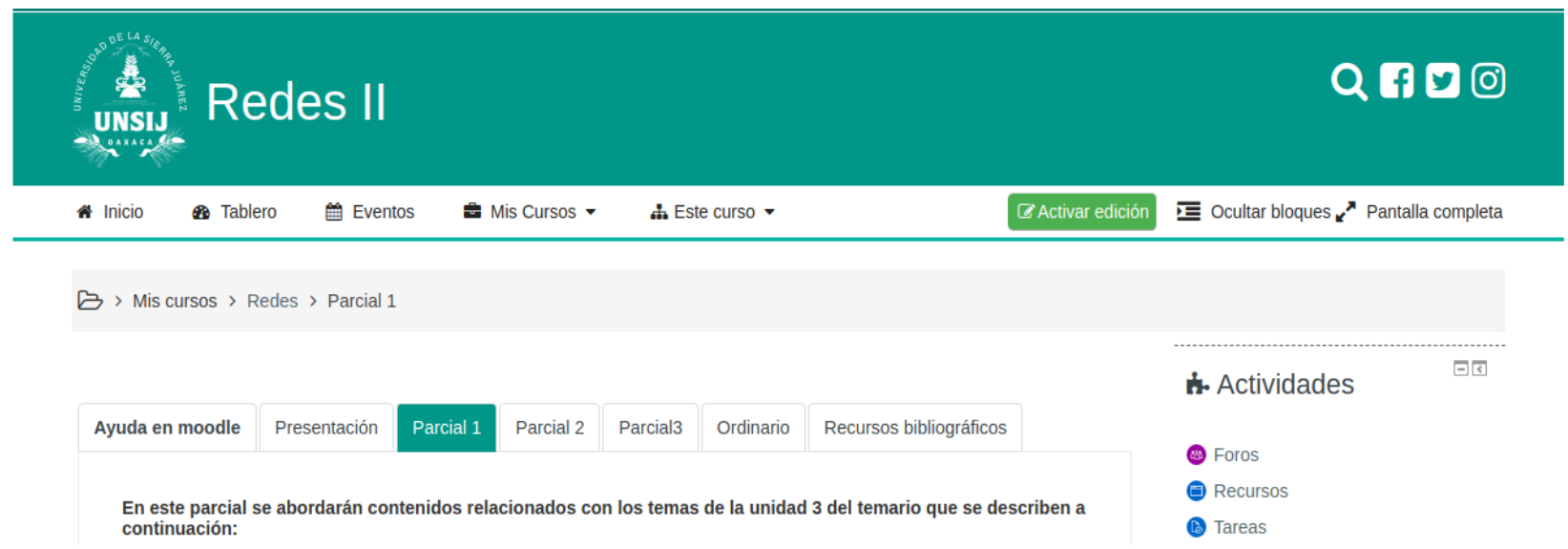

Fuente: Elaboración propia

Estos recursos de e-learning sirvieron de apoyo en las sesiones de clases presenciales, las cuales, de acuerdo con los reglamentos universitarios, deben dictarse en un horario establecido en un salón de clases o laboratorio físico. Esta combinación permitió configurar el ambiente de aprendizaje blended learning: las ventajas de la enseñanza presencial con los recursos e-learning. Así, en conjunto, brindaron la posibilidad de diseñar y llevar a cabo la planeación del proceso de enseñanza-aprendizaje con apoyo de un LMS que proporcionó diversas herramientas de la web, como los hipervínculos para relacionar las actividades de aprendizaje con las instrucciones, los contenidos y los instrumentos de evaluación, lo cual permitió al alumno seguir un orden en el desarrollo de los temas en periodos bien definidos, además de tener la posibilidad de autodirigir su aprendizaje de 


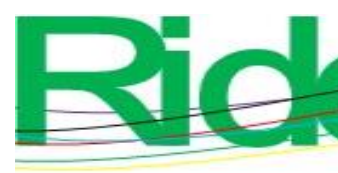

Revista Iberoamericana para la Investigación y el Desarrollo Educativo ISSN $2007-7467$

Estos aspectos observados, si bien favorecen el uso de las TIC en el proceso de enseñanza-aprendizaje, también representan un reto: lograr concienciar tanto a la directiva como al personal docente sobre la necesidad de transitar hacia un enfoque de enseñanza que permita configurar un entorno de aprendizaje enriquecido con tecnología para fortalecer la práctica docente, ya que, como se puede ver, los alumnos entrevistados tienen acceso y hacen uso de la tecnología digital en su proceso de formación, por lo que es necesario apoyar al estudiante en el desarrollo de nuevas formas de aprendizaje, y ser críticos y reflexivos sobre las fuentes informativas. Planteamientos que se pueden ver en los fines de los diferentes marcos de competencias digitales docentes: Unesco (2019), Redecker (2017) y el Intef (2017).

Dadas las circunstancias halladas en la fase del diagnóstico, el modelo que aquí se presenta resulta oportuno y cumple con lo requerido por Vaughan (2013) y Wang (2008) para poder adoptar de forma reflexiva el uso de las TIC en los entornos educativos de enseñanza presencial. Sin embargo, nuevamente, el principal reto sigue siendo el cambio de visión de los encargados de poner en práctica los principios aquí descritos. Sin duda, es necesario fomentar el desarrollo de actividades que favorezcan la presencia social, la presencia cognitiva y la presencia docente.

\section{Conclusiones}

En este trabajo se alcanza la conjugación de diversas bases metodológicas de diseño y planeación educativa, guiadas por la IBD como metodología de investigación científica. Del mismo modo, el diseño se propone con una base teórica constructivista para obtener y poner a prueba un modelo de ambiente de aprendizaje blended learning adecuado a un espacio educativo predeterminado. El punto de partida fue el análisis del contexto luego se procedió al diseño del ambiente, la puesta a prueba de este y la generación de la documentación que aquí se comparte para aportar al conocimiento de esta modalidad que mezcla las ventajas de la enseñanza presencial con la apoyada en entornos virtuales. Los resultados alcanzados aquí se siguen evaluando para seguir mejorando el diseño, sin embargo, en principio se podrían destacar algunos de los alcances con los que se cierra esta edición. 

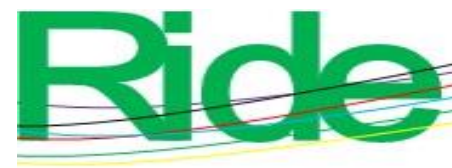

Revista Iberoamericana para la Investigación y el Desarrollo Educativo ISSN $2007-7467$

Respecto a la infraestructura de TIC, se observa que, a pesar de que la institución cuenta con ella y que existe un nivel adecuado de acceso a estos recursos, aún falta impulsar la apropiación de estas herramientas en los miembros de esta comunidad universitaria, algo que se visualiza como viable con la modalidad blended learning, ya que no solo se enfoca en la parte tecnológica, sino que permite hacer uso de los recursos de las TIC para impulsar cambios en la modalidad de la enseñanza, para lograr que los estudiantes asuman un papel activo en su proceso de aprendizaje, que es uno de los principales planteamientos de la corriente constructivista. Con respecto a las competencias digitales docentes, al igual que en el caso anterior, se observa que aún existe la posibilidad de mejorar en el conocimiento y uso de algunas áreas. Es posible afirmar que la implementación de este ambiente motiva la adopción y desarrollo de este rubro, pues es necesario echar mano de las opciones que ofrece la plataforma LMS para el despliegue del contenido pedagógico digital, aunado también al impulso que se puede dar al trabajo colaborativo para formar una comunidad virtual de enseñanza aprendizaje. Por último, se confirma que el solo uso de las TIC en la docencia no significa innovación educativa; hace falta que esta inclusión se acompañe de un conjunto de acciones que permita una transformación de los métodos de enseñanza, con un enfoque de estudiantes activos en su proceso de aprendizaje, donde la actitud de cambio del profesor juega un rol relevante. 


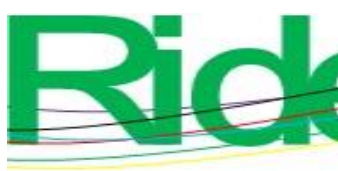
Revista Iberoamericana para la
Investigación y el Desarrollo Educativo
ISSN $2007-7467$

\section{Referencias}

Aiello, M., \& Willem, C. (2004). El blended learning como Práctica Transformadora. PixelBit. Revista de Medios y Educación, 23, 21-26. Recuperado de https://idus.us.es/bitstream/handle/11441/45580/file_1.pdf?sequence=1\&isAllowed $=\mathrm{y}$.

Asociación Nacional de Universidades e Instituciones de Educación [Anuies]. (2000). La Educación Superior en el Siglo XXI. Ciudad de México, México: Asociación Nacional de Universidades e Instituciones de Educación.

Aristizábal, D. y Dieste, B. (2015). Trabajo cooperativo y competencias transversales: una experiencia de la web 2.0 aplicada a la asignatura de educación social e intercultural (grado de maestro de primaria) en la facultad de educación. Universidad de Zaragoza. RIDE Revista Iberoamericana para la Investigación y el Desarrollo Educativo, 2(4), 34. Recuperado de https://doi.org/10.23913/ride.v2i4.46.

Ausubel, D. (2000). The Acquisition and Retention of Knowledge: A Cognitive View. U.S.: Springer.

Chadwick, C. B. (2001). La psicología de aprendizaje del enfoque constructivista. Revista Latinoamericana de Estudios Educativos, 31(4),111-126. Recuperado de https://www.redalyc.org/pdf/270/27031405.pdf.

Cher, L. and Libing, W. (eds.) (2016). Blended Learning for Quality Higher Education. Paris, France: United Nations Educational, Scientific and Cultural Organization.

Choudhury, N. (2014). World Wide Web and Its Journey from Web 1.0 to Web 4.0. International Journal of Computer Science and Information Technologies, 5(6), 8096-8100. Recuperado de http://citeseerx.ist.psu.edu/viewdoc/download?doi=10.1.1.666.6445\&rep=rep1\&typ $\mathrm{e}=$ pdf.

Cisco Systems. (30 de Agosto de 2019). Cisco Networking Academy. Obtenido de https://www.netacad.com/es

de Benito, B. y Salinas, J. M. (2016). La investigación basada en diseño en tecnología educativa. Revista Interuniversitaria de Investigación en Tecnología Educativa, (0), 44-59. Recuperado de https://doi.org/10.6018/riite2016/260631.

Delors, J. (1996). La educación encierra un tesoro. Informe a la UNESCO de la Comisión Internacional sobre la Educación para el Siglo XXI. París, Francia: Ediciones Unesco. 


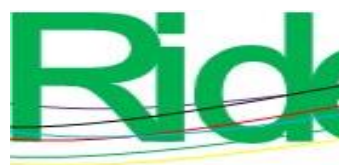

Revista Iberoamericana para la
Investigación y el Desarrollo Educativo
ISSN $2007-7467$

Escontrela Mao, R. (2008). Hacia un modelo integrador en el uso de las TIC en la educación a distancia. Apuntes y comentarios desde la investigación y la experiencia. Revista de investigación, 32(65), 15-31. Recuperado de https://dialnet.unirioja.es/servlet/articulo?codigo=2799199

Evans, D. (2011). Internet de las cosas. Cómo la próxima evolución de Internet lo cambia todo. (informe técnico). Cisco Internet Business Solutions Group (IBSG), San José. Recuperado de https://www.cisco.com/c/dam/global/es_mx/solutions/executive/assets/pdf/internetof-things-iot-ibsg.pdf.

Florez, R. (1997). Hacia una pedagogía del conocimiento. Colombia: Mc Graw Hill.

Garrison, D. R. and Kanuka, H. (2004). Blended learning: Uncovering its transformative potential in higher education. The Internet and Higher Education, 7(2), 95-105. Retrieved from https://doi.org/10.1016/j.iheduc.2004.02.001.

Garrison, R. and Vaughan, N. (2008). Blended Learning in Higher Education: Framework, Principles, and Guidelines. San Francisco, United States: Wiley and Sons.

Graham, C. (2006). Introduction to Blended Learning. In Bonk, C. J. and Graham, C. R., The Handbook of Blended Learning (pp. 1-20). United States: Jossey-Bass Inc.

Güzer, B. and Caner, H. (2013). The Past, Present and Future of Blended Learning: An in Depth Analysis of Literature. Paper presented at the $5^{\text {th }}$ World Conference on Educational Sciences. Rome, February 6-8, 2013. Retrieved from https://www.sciencedirect.com/science/article/pii/S187704281401009X\#aep-articlefootnote-id7.

Instituto Nacional de Tecnologías Educativas y Formación del Profesorado [Intef]. (2017). Marco común de competencia digital docente. Gobierno de España: Instituto Nacional de Tecnologías Educativas y Formación del Profesorado. Recuperado de https://aprende.intef.es/sites/default/files/2018-05/2017_1020_MarcoCom\%C3\%BAn-de-Competencia-Digital-Docente.pdf.

Intel Corporation. (2011). Designing Blended Learning. United Retrieved from https://educate.intel.com/download/K12/elements/blend_html/index.htm\#.

Moodle. (2019). Acerca de Moodle. Recuperado de https://docs.moodle.org/all/es/Acerca_de_Moodle. 


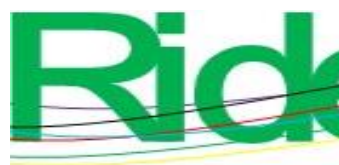

Revista Iberoamericana para la
Investigación y el Desarrollo Educativo
ISSN $2007-7467$

Necuzzi, C. (2013). Estado del arte sobre el desarrollo cognitivo involucrado en los procesos de aprendizaje y enseñanza con integración de las TIC. Argentina: Fondo de las Naciones Unidas para la Infancia.

Organización para la Cooperación y el Desarrollo Económicos [OCDE]. (2016). Programa para la Evaluación Internacional de alumnos (PISA). PISA 2015 - Resultados. Nota país.

Organización de las Naciones Unidas para la Educación, la Ciencia y la Cultura [Unesco]. (2014). Informe sobre tendencias sociales y educativas en América Latina, 2014: políticas TIC en los sistemas educativos de América Latina. Buenos Aires, Argentina: Organización de las Naciones Unidas para la Educación, la Ciencia y la Cultura / Organización de Estados Iberoamericanos para la Educación, la Ciencia y la Cultura. Recuperado de https://unesdoc.unesco.org/ark:/48223/pf0000230080.

Organización de las Naciones Unidas para la Educación, la Ciencia y la Cultura [Unesco]. (2019). Marco de competencias de los docentes en materia de TIC. Zaltbommel, Países Bajos: Van Haren Publishing.

Redecker, C. (2017). European Framework for the Digital Competence of Educators. Luxembourg: Publications Office of the European Union.

Reeves, T. C. (2000). Enhancing the Worth of Instructional Technology Research through "Design Experiments" and Other Development Research Strategies. Paper presented at the Annual Meeting of the American Educational Research Association. New Orleans, April 27, 2000.

Rodríguez, R. y Espinoza, L. A. (2017). La modalidad B-learning como alternativa de un ambiente de aprendizaje innovador. RECI Revista Iberoamericana de las Ciencias Computacionales e Informática, 6(11), 55-75. Recuperado de https://doi.org/10.23913/reci.v6i11.60.

Salgado, E. (2014). Manual de docencia universitaria. Introducción al constructivismo en la educación superior. Editorial Académica Española.

Salinas, J. (2004). Innovación docente y uso de las TIC en la enseñanza universitaria. RUSC. Revista Universidad y Sociedad del Conocimiento, 1(1), 1-16. Recuperado de https://doi.org/10.7238/rusc.v1i1.228.

Seara, M. (2010). Un nuevo modelo de universidad. Universidades para el desarrollo (2. ${ }^{\text {a }}$ ed.). Huajuapan de León, México: Universidad Tecnológica de la Mixteca. 

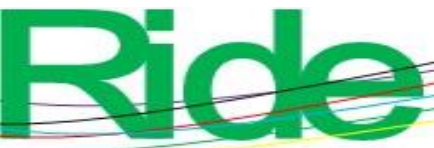

Revista Iberoamericana para la Investigación y el Desarrollo Educativo ISSN $2007-7467$

Siemens, G., Gašević, D. and Shane, D. (2015). Preparing for the Digital University: A Review of the History and Current State of Distance, Blended, and Online Learning. United States: Bill y Melinda Gates Foundation.

Tejedor, F. J., García, A. y Prada, S. (2009). Medida de actitudes del profesorado universitario hacia la integración de las TIC. Comunicar, 17(33), 115-124. Recuperado de https://doi.org/10.3916/c33-2009-03-002.

Tourón, J., Martín, D., Navarro, E., Pradas, S. e Íñigo, V. (2018). Validación de constructo de un instrumento para medir la competencia digital docente de los profesores (CDD). Revista Española de Pedagogía, 76(269), 25-54. Recuperado de https://doi.org/10.22550/rep76-1-2018-02.

Wang, Q. (2008). A generic model for guiding the integration of ICT into teaching and learning. Innovations in Education and Teaching International, 45(4), 411-419. Retrieved from https://doi.org/10.1080/14703290802377307. 


\begin{tabular}{|c|c|c|}
\hline Rol de Contribución & Autor (es) & \\
\hline Conceptualización & $\begin{array}{l}\text { Florentino Mendez Gijon } \\
\text { Magda Concepción Morales Barrera }\end{array}$ & $\begin{array}{l}\text { Igual } \\
\text { Igual }\end{array}$ \\
\hline Metodología & $\begin{array}{l}\text { Florentino Mendez Gijon } \\
\text { Magda Concepción Morales Barrera }\end{array}$ & $\begin{array}{l}\text { Igual } \\
\text { Igual }\end{array}$ \\
\hline Software & Florentino Mendez Gijon Principal & \\
\hline Validación & $\begin{array}{l}\text { Florentino Mendez Gijon } \\
\text { Magda Concepción Morales Barrera }\end{array}$ & $\begin{array}{l}\text { Principal } \\
\text { Que apoya }\end{array}$ \\
\hline Análisis Formal & $\begin{array}{l}\text { Florentino Mendez Gijon } \\
\text { Magda Concepción Morales Barrera }\end{array}$ & $\begin{array}{l}\text { Principal } \\
\text { Que apoya }\end{array}$ \\
\hline Investigación & Florentino Mendez Gijon Principal & \\
\hline Recursos & $\begin{array}{l}\text { Florentino Mendez Gijon } \\
\text { Magda Concepción Morales Barrera }\end{array}$ & $\begin{array}{l}\text { Principal } \\
\text { Que apoya }\end{array}$ \\
\hline Curación de datos & $\begin{array}{l}\text { Florentino Mendez Gijon } \\
\text { Magda Concepción Morales Barrera }\end{array}$ & $\begin{array}{l}\text { Principal } \\
\text { Que apoya }\end{array}$ \\
\hline $\begin{array}{l}\text { Escritura - Preparación del } \\
\text { borrador original }\end{array}$ & $\begin{array}{l}\text { Florentino Mendez Gijon } \\
\text { Magda Concepción Morales Barrera }\end{array}$ & $\begin{array}{l}\text { Principal } \\
\text { Que apoya }\end{array}$ \\
\hline $\begin{array}{l}\text { Escritura - Revisión y } \\
\text { edición }\end{array}$ & $\begin{array}{l}\text { Florentino Mendez Gijon } \\
\text { Magda Concepción Morales Barrera }\end{array}$ & $\begin{array}{l}\text { Principal } \\
\text { Que apoya }\end{array}$ \\
\hline Visualización & $\begin{array}{l}\text { Florentino Mendez Gijon } \\
\text { Magda Concepción Morales Barrera }\end{array}$ & $\begin{array}{l}\text { Principal } \\
\text { Que apoya }\end{array}$ \\
\hline Supervisión & Magda Concepción Morales Barrera & Principal \\
\hline $\begin{array}{l}\text { Administración de } \\
\text { Proyectos }\end{array}$ & $\begin{array}{l}\text { Florentino Mendez Gijon } \\
\text { Magda Concepción Morales Barrera }\end{array}$ & $\begin{array}{l}\text { Principal } \\
\text { Que apoya }\end{array}$ \\
\hline Adquisición de fondos & $\begin{array}{l}\text { Florentino Mendez Gijon } \\
\text { Magda Concepción Morales Barrera }\end{array}$ & $\begin{array}{l}\text { Principal } \\
\text { Que apoya }\end{array}$ \\
\hline
\end{tabular}

